\section{Identification of Myomaker in Yellowfin Seabream (Acanthopagrus latus) (Hottuyn, 1782) and its Transcriptional Regulation by Two MyoDs}

Kecheng Zhu ${ }^{1,2,3}$, Peiying He ${ }^{1}$, Baosuo Liu ${ }^{1,2,3}$, Huayang Guo ${ }^{1,2,3}$, Nan Zhang ${ }^{1,2,3}$, Liang Guo ${ }^{1,2,3}$, Shigui Jiang ${ }^{1,2,3}$ and Dianchang Zhang ${ }^{1,2,3, *}$

${ }^{1}$ Key Laboratory of South China Sea Fishery Resources Exploitation and Utilization, Ministry of Agriculture and Rural Affairs; South China Sea Fisheries Research Institute, Chinese Academy of Fishery Sciences, 510300, Guangzhou, Guangdong Province, PR China

${ }^{2}$ Guangdong Provincial Engineer Technology Research Center of Marine Biological Seed Industry, Guangzhou, Guangdong Province, PR China

${ }^{3}$ Guangdong Provincial Key Laboratory of Fishery Ecology and Environment, Guangzhou, Guangdong Province, PR China

\begin{abstract}
A B S T R A C T
Myomaker is a muscle-specific membrane protein that is essential for myoblast fusion. Myomaker is regulated by myoblast determination protein $(\mathrm{MyoD})$, a muscle-specific basic helix-loop-helix (bHLH) transcription factor in higher vertebrates. However, the transcriptional regulatory mechanism of the myomaker gene has not been explored in marine fishes. In the present study, molecular cloning, bioinformatic analysis and transcriptional analysis of Acanthopagrus latus myomaker (Almyomaker) were performed. The open reading frame (ORF) sequence of Almyomaker is $858 \mathrm{bp}$, which encodes a polypeptide of 285 amino acids. Moreover, phylogenetic and gene structure analysis indicates that Almyomaker is highly conserved among vertebrates. The tissue distribution pattern shows that Almyomaker is more highly expressed in white muscle than in other tissues. Furthermore, to explore whether two MyoDs are modulators of Almyomaker, a promoter analysis was performed using progressive deletion mutations of Almyomaker. The results of promoter activity assays show that Almyomaker expression is notably activated by two MyoDs. Transcriptional activity of the Almyomaker promoter was observed to dramatically decrease after targeted mutation of the MyoD1 M1 and MyoD2 M2 binding sites. In summary, MyoD1 and MyoD2 play an important role in the regulation of Almyomaker expression and may promote myoblast fusion during muscle development and growth by modulating Almyomaker expression.
\end{abstract}

Article Information
Received 16 January 2020
Revised 14 February 2020
Accepted 04 March 2020
Available online 24 December 2020
Authors' Contribution
KCZ, SGJ and DCZ designed the
research and wrote the paper. PYH
and KCZ performed the research.
HYG and NZ analyzed the data.
BSL and LG contributed reagents/
materials/analysis tools.
Key words
Acanthopagrus latus, Myomaker,
MyoD, Promoter activity, Mutation
analyses.

\section{INTRODUCTION}

$\mathrm{T}$ he fusion of myoblasts is an important process to generate multinucleated myofibers during skeletal muscle regeneration and development (Kim et al., 2015). To date, several proteins, such as myoferlin (Doherty et al., 2005), myogenin and myoD (Tapscott, 2005), Myocyte enhancer factor $2 \mathrm{~s}$ (mef2s) (Hinits and Hughes, 2007), Rasrelated C3 botulinum toxin substrate 1 (Rac1) (Vasyutina et al., 2009), nephrin (Sohn et al., 2009), Junctional adhesion molecule b and c (Jamb and Jamc) (Powell and Wright, 2011; Shi et al., 2019), CD9 (Charrin et al., 2013), and CD81 (Charrin et al., 2013), involved in the process

\footnotetext{
* Corresponding author: zhangdch@scsfri.ac.cn 0030-9923/2021/0001-0227 \$9.00/0

Copyright 2021 Zoological Society of Pakistan
}

of muscle development, have been already identified. However, myomaker, also called transmembrane protein $8 \mathrm{c}$ (TMEM8c), is a muscle-specific protein that is absolutely indispensable for myoblast fusion and sufficient to promote fibroblast fusion with muscle cells in zebrafish (Danio rerio), mice (Mus musculus), and chickens (Gallus gallus) (Millay et al., 2013, 2014, 2016; Landemaine et al., 2014; Luo et al., 2015; Zhang et al., 2017). Knockdown of myomaker in zebrafish, mice, and chickens indicates that myomaker is necessary for myoblast fusion and that loss of myomaker function causes abnormal muscle development (Millay et al., 2013, 2014; Landemaine et al., 2014; Luo et al., 2015; Shi et al., 2018). The amino acid (aa) sequence of myomaker is highly conserved throughout vertebrate species (Millay et al., 2013), and its function in myogenesis is also conserved between mammals and fish (Landemaine et al., 2014). Myomaker is a hydrophobic protein 
consisting of 221 aa that localizes to intracellular vesicles and the plasma membrane in skeletal myocytes (Millay et al., 2013). The structural features of myomaker span the bilayer seven times with an extracellular N-terminal region and cytosolic C-terminal tail with unknown conserved functional domains (Millay et al., 2016).

Myogenin (MyoG) and MyoD, members of myogenic regulatory factors (MRFs), are pivotal transcription factors (TFs) in myogenesis and can control the transcription of most muscle-related genes (Braun et al., 1989; Edmondson and Olson, 1990; Berkes and Tapscott, 2005; Braun and Gautel, 2011). The two MRFs play a critical role in the regulation of myoblast differentiation. The transcription of myomaker is mediated through the activity of musclespecific TFs MyoG and MyoD (Luo et al. 2015; Quinn et al., 2017; Zhang et al., 2017; Ganassi et al., 2018). Additionally, during the acute and chronic muscle regeneration process, MyoD can induce myomaker expression in muscle satellite cells and accelerate myoblast fusion. Then, the expression of myomaker decreased rapidly after fusion (Millay et al., 2014; Landemaine et al., 2014; Demonbreun et al., 2015; Zhang and Roy, 2017). In the MyoD signalling pathway, a downstream gene, myomixer, is activated by MyoD. Nonmyoblasts can fuse into multicellular cells when myomaker and myomixer are overexpressed (Bi et al., 2017; Quinn et al., 2017; Zhang et al., 2017). In avians, the essential role of myomaker is in myoblast fusion and shows that MyoD can regulate myomaker expression (Luo et al., 2015). Two highly conserved e-box mutations in the 5 '-regulatory region sequence of the myomaker gene in mice and chickens have been verified, suggesting that the importance of the e-box is in regulating the transcription and expression of myomaker (Millay et al., 2014; Luo et al., 2015). Moreover, when two MyoD binding sites of the myomaker promoter are deleted in chickens, its promoter activity is significantly reduced, indicating that MyoDs have a positive regulatory effect on myomaker (Luo et al., 2015). The C-terminal region of myomaker is essential for the function of three cysteine residues, which are speculated to be palmitoylated (Millay et al., 2016). Myomaker displays N-terminal glycine predicted to sustain myristoylation (Bologna et al., 2004). However, the cellular mechanism of myomaker and the regulatory mechanism of its expression during myogenesis have not been determined in marine fish.

Yellowfin seabream (Acanthopagrus latus) (Hottuyn, 1782), Sparidae, and Perciformes are considered significant aquaculture fish in southern China due to their economic value. Nevertheless, the muscle growth rate is too low in A. latus. Consequently, this fish is known as a specific model for exploring regulatory mechanisms in muscle development in marine fish. In the present study, to investigate the underlying function of Almyomaker and transcriptional regulation of two AlMyoDs, this study focused on illuminating the consequence of MyoD in the activation of Almyomaker expression. A musclespecific membrane protein gene from A. latus, myomaker (Almyomaker), was confirmed. Promoter activity assays via the mutation of potential MyoD binding sites are executed to determine key elements in the Almyomaker candidate sequence. The present study may contribute to further exploration of myomaker function in marine fish and help to elucidate the regulatory mechanism for myoblast fusion.

\section{MATERIALS AND METHODS}

\section{Animals and tissues collection}

Fish (body weight: $289 \pm 18.5 \mathrm{~g}$ ) were collected from Yangjiang Marine Fish Farm in Guangdong Province, China. For the present study, three healthy fish were used, and 13 tissues (heart, male gonad, eye, skin, brain, fin, spleen, small intestine, gill, white muscle, kidney, liver, and stomach) were isolated to analyze the myomaker expression level in different tissues. Then, the flash was frozen in liquid nitrogen and stored at $-80^{\circ} \mathrm{C}$ until use.

\section{Gene cloning and bioinformatics of myomaker}

Total RNA $(1 \mu \mathrm{g})$ was extracted from $A$. latus white muscle by TRIzol Reagent (Takara, Japan). To synthesize cDNA, the Prime ScriptTM RT reagent Kit (Takara) was used according to the manufacturer's instructions. Putative myomaker, MyoD1 and MyoD2 sequences were acquired according to genomic data of $A$. latus (Sequence Read Archive under BioProject PRJNA566024). To verify the accuracy of the derived sequence, gene-specific primers were designed (Table I). The PCR system (volume) and procedure employed were described previously (Zhu et al., 2014). The amplified products, linked into the pEASY-T1 vector (TransGen Biotech, China), were purified by a DNA Purification Kit (Tiangen, China) and then sequenced (Invitrogen, China). Confirmed recombinants were transformed into competent Trans1-T1 cells (TransGen Biotech, China). A BLAST search on the supposed myomaker open reading frame (ORF) sequence further confirmed the accuracy and validity of the analysis.

The derived aa sequence from the cloned Almyomaker ORF was aligned with other myomaker orthologue proteins from the NCBI and Ensembl databases (Fig. 2). Multiple sequence alignment was performed by ClustalX version 2.0 (Larkin et al., 2007) with default parameters. Phylogenetic analyses for all myomaker aa sequences were achieved using MEGA 6.0 (Tamura et al., 2013) with maximum likelihood (ML) methods (LG + G 
model, bootstrap 1000). All available myomaker genome sequences were obtained from Ensembl (http://asia. ensembl.org/) and Genome Browser (http://genome.ucsc. edu/cgi-bin/hgBlat). To predict the signal peptides and transmembrane domain, the Signal P3.0 server (http:// www.cbs.dtu.dk/services/SignalP/), TMHMM (http:// www.cbs.dtu.dk/services/TMHMM/), and SMART (http:// smart.embl-heidelberg.de/) were used. Moreover, the genome structure and phylogenetic tree were embellished using Adobe PhotoShop CS6 (Adobe, San Jose, CA) and FigTree v1.4.2 (http://tree.bio.ed.ac.uk/software/figtree/), respectively.

Table I.- Primers used for sequence cloning, deletion mutant construction, and qRT-PCR.

\begin{tabular}{|c|c|}
\hline $\begin{array}{l}\text { Subject and } \\
\text { Primers }\end{array}$ & $\begin{array}{c}\text { Nucleotide } \\
\text { sequence }\end{array}$ \\
\hline \multicolumn{2}{|c|}{ Primers for sequence cloning } \\
\hline \multicolumn{2}{|c|}{ Myomaker-ORF F: ATGGGTGCGTTTATTGCCAAGAT } \\
\hline \multicolumn{2}{|c|}{ Myomaker-ORF R: TCACTTCCAGCCATTCATCT } \\
\hline \multicolumn{2}{|c|}{ MyoD1-ORF-F CGCGGATCCATGGAGCTGTCG } \\
\hline \multicolumn{2}{|c|}{ MyoD1-ORF-R C } \\
\hline \multicolumn{2}{|l|}{ MyoD2-ORF-F } \\
\hline \multicolumn{2}{|c|}{ MyoD2-ORF-R CCGCTCGAGTCAGAGCGGCTCCTGGATGCT } \\
\hline \multicolumn{2}{|c|}{ Deletion mutant construction } \\
\hline \multicolumn{2}{|c|}{ Myomaker-pF1 CGGGGTACCC } \\
\hline \multicolumn{2}{|c|}{ Myomaker-pF2 CGGGGTACCCGCC } \\
\hline \multicolumn{2}{|l|}{ Myomaker-pF3 } \\
\hline \multicolumn{2}{|l|}{ Myomaker-pF4 } \\
\hline \multicolumn{2}{|c|}{ Myomaker-pF5 CGGGGTACCAAACTTTAAAGGGCCAAGCTG } \\
\hline \multicolumn{2}{|c|}{ Myomaker-pR CCGCTCGAGCATGGTGGAGGGCCAGTAGACA } \\
\hline \multicolumn{2}{|c|}{ Primers for qRT-PCR } \\
\hline Myomaker-F & CTGGGCGACTTTGATGAGC \\
\hline Myomaker-R & AGTGATGATGAAGACAGCGGA \\
\hline $\mathrm{EF} 1 \alpha-\mathrm{F}$ & AAGCCAGGTATGGTTGTCAACTTT \\
\hline EF1 $\alpha-R$ & CGTGGTGCATCTCCACAGACT \\
\hline
\end{tabular}

\section{Real-time quantitative PCR analysis}

Specific primers for real-time quantitative PCR (qRTPCR) were designed by Primer Premier 5.0 (Premier Biosoft, USA) based on cloned nucleotide sequences (Table I) (Zhu et al., 2020). The myomaker, MyoDs and elongation factor 1-alpha $(E F-1 \alpha)$ were tested and used as a target and reference gene, respectively. qRTPCR was implemented in a quantitative thermal cycle (Mastercycler ${ }^{\circledR}$ eprealplex; Eppendorf, Germany). The PCR system (volume) was described above. The qRTPCR program was conducted as follows: $95^{\circ} \mathrm{C}$ for $2 \mathrm{~min}$ followed by 40 cycles of $95^{\circ} \mathrm{C}$ for $10 \mathrm{~s}, 56^{\circ} \mathrm{C}$ for $10 \mathrm{~s}$, and $72^{\circ} \mathrm{C}$ for $20 \mathrm{~s}$. Gene expression was determined using the
$2^{-\Delta \Delta C t}$ method (Livak et al., 2001).

Plasmid construction, cell culture and dual-luciferase reporter assays

Briefly, to clone the target fragment of Almyomaker, genomic DNA was isolated from the white muscle of $A$. latus, as previously described in other marine fish (Sun et al., 2013). The ORF sequence upstream of the myomaker gene was obtained from genomic data of A. latus. Moreover, to obtain the recombinants of $M y o D 1$ and $M y o D 2$, the integrated sequences of $M y o D 1$ and $M y o D 2$ ORFs were inserted into the pCDNA3.1-Flag vector (Invitrogen, USA) using specific primers (Table I) (Zhu et al., 2020). To confirm the effect of AlMyoD1 and AlMyoD2 on Almyomaker expression, five different truncated regions from Almyomaker were amplified by peculiar primers with $K p n \mathrm{I}$ and $X h o \mathrm{I}$ restriction sites (Table I). The forward primers (Myomaker-pF1, Myomaker-pF2, MyomakerpF3, Myomaker-pF4, and Myomaker-pF5) were designed with a 5' KpnI site, and the common reverse primer (Myomaker-pR) was designed with a 3' XhoI site (Table I). These primers were used to acquire the target region (Myomaker-pF1, 2,090 bp) and four truncated fragments ((i) Myomaker-pF2, 1,483 bp; (ii) Myomaker-pF3, 1,211 bp; (iii) Myomaker-pF4, 436 bp and (iv) Myomaker-pF5, 302 bp) (Fig. 5). PrimeSTAR Master Mix (Takara, Japan) was used to amplify the five truncated mutants. The PCR program consisted of $95^{\circ} \mathrm{C}$ for $4 \mathrm{~min}$, followed by 30 cycles of $95^{\circ} \mathrm{C}$ for $40 \mathrm{~s}, 56^{\circ} \mathrm{C}$ for $40 \mathrm{~s}$, and $72^{\circ} \mathrm{C}$ for 1 min. Subsequently, the PCR products were purified by the general DNA Purification Kit (Tiangen, China). A pGL3basic (Promega, USA) vector and purified PCR products were digested with $K p n \mathrm{I}$ and $\mathrm{XhoI}$ and linked by T4 DNA ligase (Takara, Japan) overnight at $16^{\circ} \mathrm{C}$. Recombined plasmids were extracted using the EndoFree Plasmid Giga Kit (Tiangen, China), and recombinants were validated by sequencing, as described above.

Furthermore, to investigate the possible function of MyoD1 or MyoD2 binding sites on the core myomaker promoter and 5'UTR sequences, three truncated mutations of recombinant plasmids were established. The TF binding site prediction (TFBS)-JASPAR database (http://jaspar. genereg.net/), TRANSFAC ${ }^{\circledR}$, and MatInspector ${ }^{\circledR}$ were used to search for potential binding sites in the myomaker sequence with MyoD1 and MyoD2, respectively. Then, according to the manufacturer's protocol, site-directed mutagenesis was conducted with a QuickChange II Site-Directed Mutagenesis Kit (Vazyme, USA) from the deletion mutant pGL3-basic-myomaker-p5, which was regarded as the wild-type plasmid. The prediction of three binding sites (M1, M2, and M3) was directly deleted, and the schematic diagram and sequences of homologous 
TF binding sites are shown in Figure 6A and Table II, respectively. The role of TF binding site mutations on the promoter activity of Almyomaker was explained by a dual luciferase assay, as described below (Genecreate, China).

Table II.- Sequences of putative binding sites on AlMyomaker-P5 sequence.

\begin{tabular}{lcc}
\hline $\begin{array}{l}\text { Putative binding } \\
\text { sites }\end{array}$ & $\begin{array}{c}\text { Nucleotide } \\
\text { sequence }\end{array}$ & $\begin{array}{c}\text { Mutated } \\
\text { pattern }\end{array}$ \\
\hline M1 & GGACAGCTGGGT & Deletion \\
M2 & GGACATCTACTT & Deletion \\
M3 & GACTGAGGAACT & Deletion \\
\hline
\end{tabular}

Human embryonic kidney (HEK293T) cells were cultured in DMEM (Gibco, USA) with 10\% fetal bovine serum (FBS) (Invitrogen, USA) accompanied by $100 \mu \mathrm{g} /$ $\mathrm{mL}$ streptomycin and $100 \mathrm{U} / \mathrm{mL}$ penicillin (Thermo Fisher Scientific, USA) at $37^{\circ} \mathrm{C}$ in a humidified incubator under $5 \% \mathrm{CO}_{2}$. The procedure of transfection and dual luciferase reporter assays were described by $\mathrm{Li}$ et al. (2017). Relative luciferase activities (the ratio of firefly and ranilla luciferase activities) were measured and calculated using the VICTOR ${ }^{\mathrm{TM}}$ X2 Multi-label Plate Reader (PerkinElmer, Inc., Waltham, MA, USA).

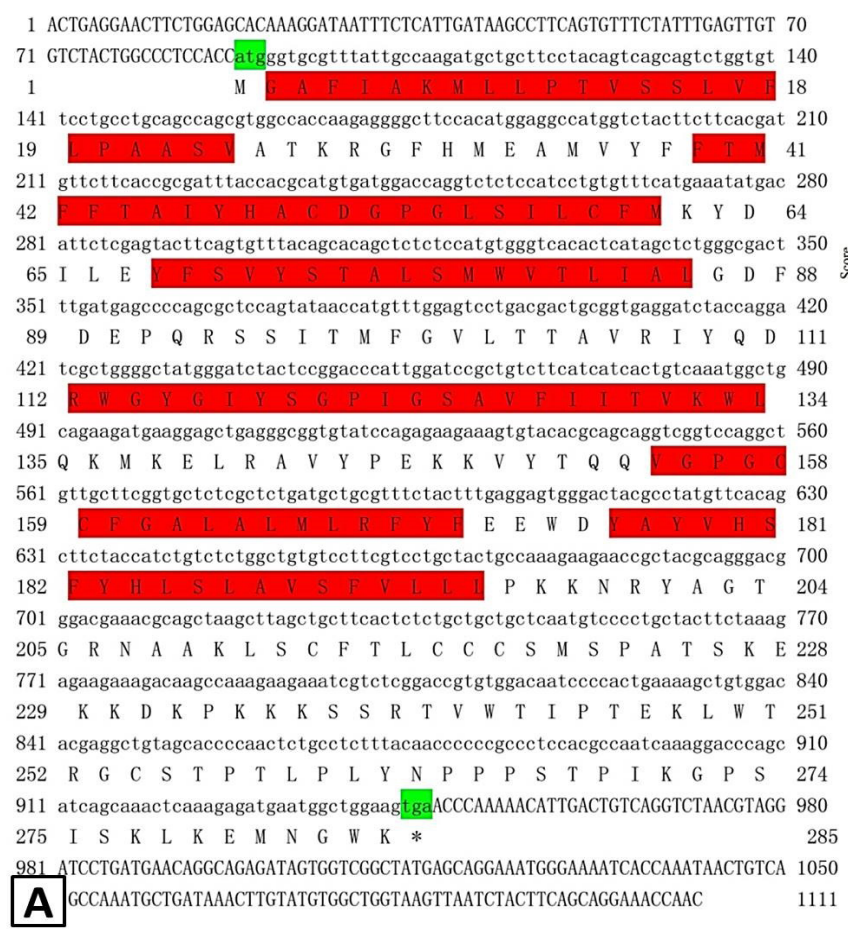

\section{Statistical analysis}

All trials were executed in triplicate in the present study. All values are shown with three replicates \pm SE. Significant differences were calculated by one way ANOVA tests. $P$ values $<0.05$ were considered to be significant.

\section{RESULTS}

\section{Sequence characterization of Almyomaker}

The genomic sequence of Almyomaker is 3,383 bp, including 6 exons and 5 introns (Supplementary Fig. S1). The full-length ORF of Almyomaker is 855 bp (Fig. 1A), encoding peptides of 285 amino acids with a predicted molecular weight of $32.14 \mathrm{kDa}$ and a theoretical isoelectric point of 9.44 (Accession No. MN266854). A conserved domain (DUF3522 superfamily starting from the $3^{\text {rd }}$ amino acid and ending at the 185th amino acid) was detected using BLAST (Huang et al., 2019). Hydrophobicity profiles show that the Almyomaker protein includes more hydrophobic amino acids than hydrophilic amino acids (Fig. 1B). The results of TMHMM indicate that there are 6 obvious transmembrane domains in the Almyomaker protein (Fig. 1C), which is consistent with the analysis of goose Anser cygnoides (He et al., 2017).
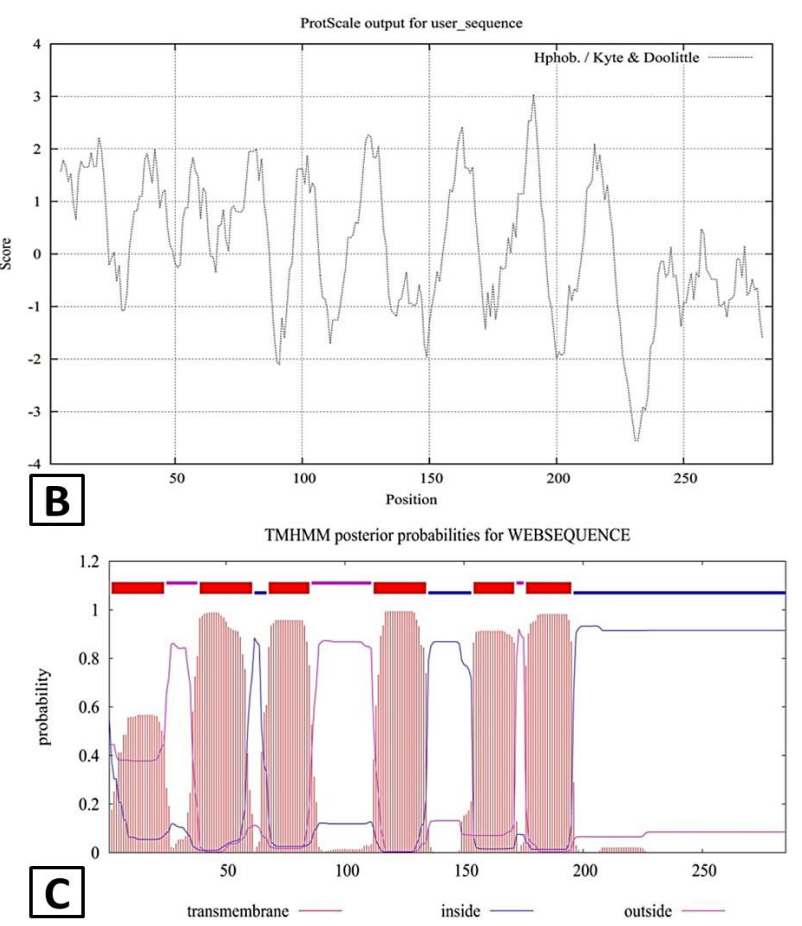

Fig. 1. Sequence characterization of the Myomaker gene in Acanthopagrus latus. A, the nucleotide sequence of the Myomaker gene and the deduced amino acid sequence of T. ovatus. Initiation and termination codons are marked by green. Six transmembrane domains are shown in red; B, hydrophobicity profile of Myomaker; C, TMHMM posterior probabilities of Myomaker. 
Pf 1 MGAFIAKMLLPTASSLVFLPAASVAAKRGFHTEAMVYFFTMFFTAIYHACDGPGLSILCFMRYDILEYFSVYGTVLSMWVTLVALGDFDEPQRSTISMFGVLTIAVRIYQDRWGYGIYSGPIGSAVFIITIKWLOKMKQLRAIYP 145 $X_{m} 1$ MGAFIAKMLLPTASSLVFLPAASVAAKRGFHTEAMVYFFTMFFTAIYHACDGPGLSVLCFMRYDILEYFSVYGTALSMWVTLVALGDFDEPQRSTISMFGVLTIGVRIYQDRWGYGIYSGPIGSAVFIITIKWLOKMKQLRAVYP 145 O1 1 MGA OI 1 MGAFAKM O1 1 MGAFAKMLLPFSLLLPTASVAAKRGF Tn 1 MGAFTAKMLLPTVSLVFLPTASVAAKRGFHMEAMYYFFTMFFAAIYHACDGPGLSILCFMRYDVLEYFSVYGTALSMWVTLIALGDFDEPQRSSLTMFGVLTIAVRIYQDRWGYGIYSGPIGSAVFIITVKWLQKMKQLRAVYP 145 Dr 1 MGAFIAKMLLPTISSLVFVPAASVAAKRGFHMEAMVYFFTMFFTAIYHACDGPGLSILCFMKYDILEYFSVYGTAISMWVTLLALGDFDEPKRSSLTMFGVLTAAVRIYQDRLGYGIYSGPIGTAVFMITVKWLOKMKEKKGLYP 145 $G_{g} 1$ MGSLVAKLLLPTLSTLVFLPTISIAAKRRFHMEAMVYFFTMFFVAIYHACDGPGLSVLCFMRYDILEYFSIYGTALSIWVSLMALAEFDEPKRSTFIMFGVLTIAVRTYHDRWGYGIYSGPIGTAVLVITVKWLOKMKEKKGLYP 145 PS 1 MGSLVAKLLLPTLSSLVFLPTISIAAKRRFHMEAMVYFFTMFFVAIYHACDGPGLSVLCFMRYDILEYFSIYGTALSIWVSLMALAEFDEPKRSTFVMFGVLTIAVRIYHDRWGYGVYSGPIGTAVLVITVKWLQKMKEKRGLYP 145 Hs 1 MGTLVAKLLLPTLSSLAFLPTVSIAAKRRFHMEAMVYLFTLFFVALHHACNGPGLSVLCFMRHDILEYFSVYGTALSMWVSLMALADFDEPKRSTFVMFGVLTIAVRIYHDRWGYGVYSGPIGTAILIIAAKWLQKMKEKKGLYP 145 Mm 1 MGTVVAKLLLPTLSSLAFLPTVSIATKRRFYMEAMVYLFTMFFVAFSHACDGPGLSVLCFMRRDILEYFSIYGTALSMWVSLMALADFDEPQRSTFTMLGVLTIAVRTFHDRWGYGVYSGPIGTATLIIAVKWLKKMKEKKGLYP 145 Lc 1 MGAIVAKLLLPTISSLVFLPAISIAAKRRFHMEAMVYFFTMFFVAIYHACDGPGLSVLCFMRYDILEYFSIYGTALSMWVTLIALAEFDEPKRSTLVIFGVLTIAVRIYQDRWGYGVYSGPIGTAVFIITVKWLKTMKEKKSLFP 145

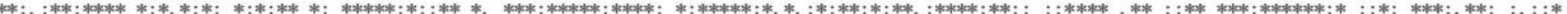

Pf 146 EKSVYTQQVGPGCCFGALALMLRFYFEEWDYAYVHSFYHLSLAVSFVLLLPKKNRYAGSGENAAKITCLTLCCCTMSPGSSKEKTNK-PQKKKSSRSVWRLPTEKLWPRGSGTPTLPVCAPPPSPPVKGTSVSKLKELLNGKK 286

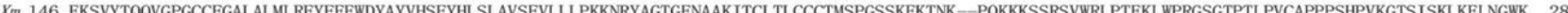
1146 TKKVYTOOVGPCCCFGALALV 28 On OI 146 ERT TI 146 EKTVYT

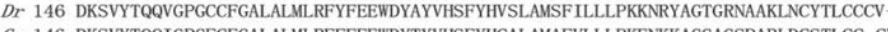
Gg 146 DKSVYTQQIGPGFCFGALALMLRFFFEWDYTYVHSFYHCALAMAFVLLLPKENKKAGGAGSPARLDCSTLCC-CVPS 146 DKSVYTQQIGPGFCFGALALMLRFFFEWDYTYVHSFYHCALAMSFVLLLPKVNKKAGNGGNPAKLDCSTLCC-CVHs 146 DKSVYTQQIGPGLCFGALALMLRFFFEDWDYTYVHSFYHCALAMSFVLLLPKVNKKAGSPGTPAKLDCSTLCCACV MIr 146 DKSIYTQQIGPGLCFGALALMLRFFFEEDYTYVHSFYHCALAMSFVLLLPKVNKKAGNAGAPAKLTFSTLCCTCV Lc 146 DRSVYAQQVGPGFCFGALALMLRFYFEEWDYAYVHSFYHCSLAMSFVLMLPKLNKQGGSGGTPAKLSCCTLCC-CY

A

\begin{tabular}{|c|c|c|c|c|c|c|c|c|c|c|c|c|c|c|}
\hline \multicolumn{12}{|c|}{ Total AA identity $\%$} & & Species & No. \\
\hline 1 & 2 & 3 & 4 & 5 & 6 & 7 & 8 & 9 & 10 & 11 & 12 & & & \\
\hline 100 & 92.0 & 87.1 & 86.7 & 86.3 & 79.7 & 71.5 & 61.0 & 59.7 & 58.7 & 56.5 & 54.7 & 1 & Acanthopagrus latus & MN266854 \\
\hline - & 100 & 89.2 & 90.3 & 88.5 & 78.1 & 73.2 & 58.0 & 60.5 & 60.1 & 63.6 & 55.0 & 2 & Oreochromis niloticus & ENSONIT00000017480.1 \\
\hline- & - & 100 & 83.9 & 97.6 & 65.3 & 69.8 & 57.0 & 59.4 & 59.1 & 56.1 & 54.7 & 3 & Xiphophorus maculatus & XP_005812520.1 \\
\hline- & - & - & 100 & 83.9 & 65.2 & 72.2 & 56.9 & 65.5 & 59.0 & 57.6 & 54.7 & 4 & Oryzias latipes & XP_004072981.1 \\
\hline - & - & - & - & 100 & 65.3 & 69.4 & 56.7 & 59.4 & 58.7 & 56.1 & 54.4 & 5 & Poecilia formosa & ENSPFOT00000029545.1 \\
\hline - & - & - & - & - & 100 & 88.6 & 77.7 & 80.9 & 79.6 & 75.1 & 72.9 & 6 & Danio rerio & ENSDART00000161578.2 \\
\hline- & - & - & - & - & - & 100 & 75.5 & 78.6 & 78.2 & 73.3 & 72.4 & 7 & Tetraodon nigroviridis & ENSTNIT00000020078.1 \\
\hline - & - & - & - & - & - & - & 100 & 83.2 & 95.0 & 86.9 & 85.5 & 8 & Gallus gallus & ENSGALT00000034784.5 \\
\hline- & - & - & - & - & - & - & - & 100 & 85.9 & 80.5 & 78.3 & 9 & Latimeria chalumnae & XP_005990668.1 \\
\hline- & - & - & - & - & - & - & - & - & 100 & 89.6 & 86.9 & 10 & Pelodiscus sinensis & ENSPSIT00000008830.1 \\
\hline B & - & - & - & - & - & - & - & - & - & 100 & 88.7 & 11 & Homo sapiens & ENST00000339996.3 \\
\hline & - & - & - & - & - & - & - & - & - & - & 100 & 12 & Mus musculus & ENSMUST00000009358.8 \\
\hline
\end{tabular}

Fig. 2. Amino acid sequences of Myomaker homologues in vertebrates. A, amino acid alignment of Myomaker proteins from A. latus (Al), Oreochromis niloticus (On), Xiphophorus maculatus (Xm), Oryzias latipes (Ol), Poecilia formosa (Pf), Danio rerio (Dr), Tetraodon nigroviridis (Tn), Gallus gallus $(\mathrm{Gg})$, Latimeria chalumnae (Lc), Pelodiscus sinensis (Ps), Homo sapiens (Hs), and Mus musculus (Mm). Conserved sequences are marked with asterisks. B, Percent identities of Myomaker amino acids compared to the above 12 Myomaker amino acids, and the accession numbers of the Myomaker sequences used are also listed.

The myomaker amino acids in A. latus reveal sequence homology with those in the other metazoan species (Fig. 2A) and are most highly homologous with Xiphophorus maculatus, Oryzias latipes, Poecilia formosa, and Tetraodon nigroviridis. Moreover, a BLAST analysis indicated that the Almyomaker protein sequence shares high sequence identity with myomaker sequences from other teleosts containing tilapia (O. niloticus, 92 $\%$ ), platyfish ( $X$. maculatus, 87.1\%), medaka (O. latipes, $86.7 \%$ ), and molly (P. formosa, 86.3\%) and low sequence identity with humans (Homo sapiens, 56.5\%) and mice (Mus musculus, 54.7\%) (Fig. 2B).

\section{Almyomaker structural and phylogenetic analyses}

The genomic structural features and phylogenetic relationship of myomaker were determined and constructed in metazoans (Fig. 3). The lengths and distributions of the exons and introns of metazoan myomaker genes are displayed in Supplementary Table I. Six exons and five introns were identified in myomaker gene sequences, 
except for zerbrafish (Danio rerio), Tetraodon (Tetraodon nigroviridis), Chinese softshell turtle (Pelodiscus sinensis), Coelacanth (Latimeria chalumnae), chicken (Gallus gallus), H. sapiens and M. musculus myomaker, which only possess five exons and four introns. Furthermore, the sizes of the exon sequences reveal that there is nearly no diversity among species. Additionally, the phylogenetic tree was constructed with the full-length myomaker aa of various fish, mammalia, aves, and amphibia species. Almyomaker is grouped together with other Perciformes, such as $O$. niloticus. The phylogenetic tree clearly shows that fish evolve into one branch followed by Amphibians,
Aves and then Mammals (Huang et al., 2019).

\section{Tissue expression of Almyomaker}

The mRNA levels of Almyomaker are determined by qRT-PCR in various kinds of tissues. qRT-PCR shows that Almyomaker is broadly distributed in various tissues, and the abundance of mRNA varies among tissues. The highest Almyomaker transcriptions were detected in the white muscle followed by the brain and spleen, whereas the lowest Almyomaker expression levels were found in the liver, skin and stomach (Fig. 4).

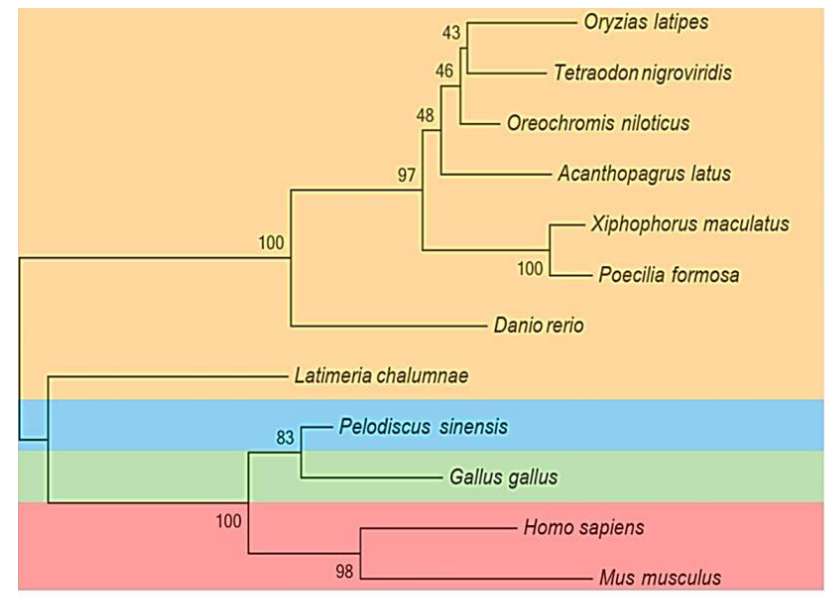

$\stackrel{\longmapsto}{\longmapsto .020}$

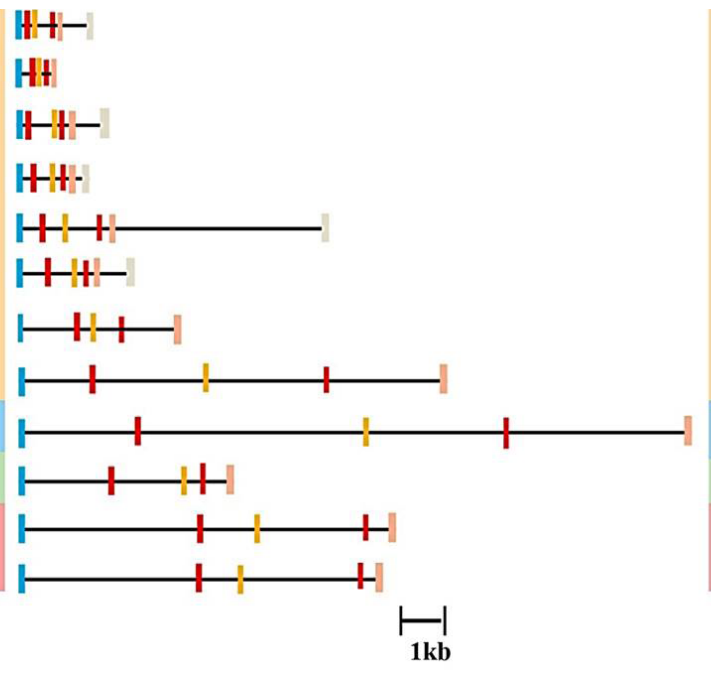

Osteichthyes

Reptilia

Aves

Mammalia

Fig. 3. Phylogenetic relationship and structure of the Almyomaker gene with other vertebrates. Genome structure analysis of Almyomaker genes according to their phylogenetic relationship. Lengths of exons and introns of each Myomaker gene are displayed proportionally. Different colour boxes and lines represent exons and introns, respectively. The identical colour boxes represent homologous sequences.

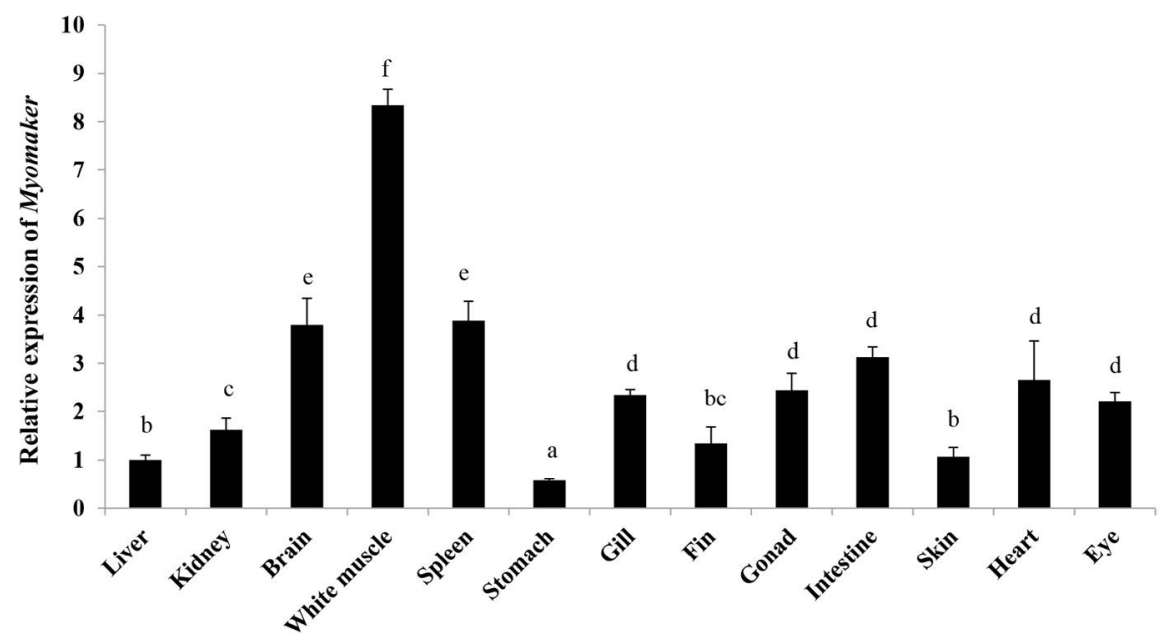

Fig. 4. Relative expression levels of Almyomaker in different tissues. The twelve tissues are heart, gonad, eye, skin, brain, fin, spleen, small intestine, gill, white muscle, kidney, liver, and stomach. Different letters indicate significant differences $(p<0.05)$. 


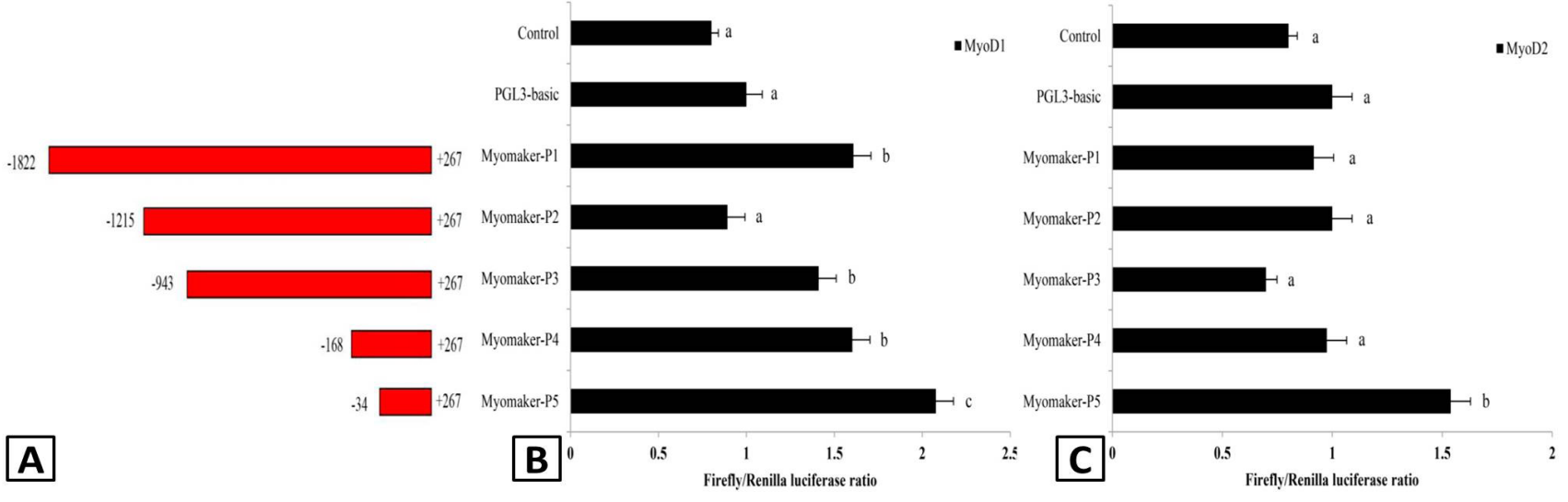

Fig. 5. Promoter activity analysis of the Almyomaker gene. A, the structure of the Almyomaker promoter and 5'UTR region. Five recombinant plasmids were denoted myomaker-p1 $(-1822$ to +267$)$, myomaker-p2 $(-1215$ to +267$)$, myomaker-p3 $(-943$ to +267$)$, myomaker-p4 (-168 to +267$)$ and myomaker-p5 $(-34$ to +267$)$. B and C, Transcriptional activity of the Almyomaker promoter. These plasmids were transfected along with the transcription factors MyoD1 (B) and MyoD2 (C) into HEK 293T cells. Dualluciferase activity was driven by the Almyomaker promoter upon the transfection of pcDNA3.1-MyoD1, pcDNA3.1-MyoD2 or pcDNA3.1 into HEK 293T cells. Data are presented as the means of three replicates \pm SE. Different letters indicate significant differences $(p<0.05)$.

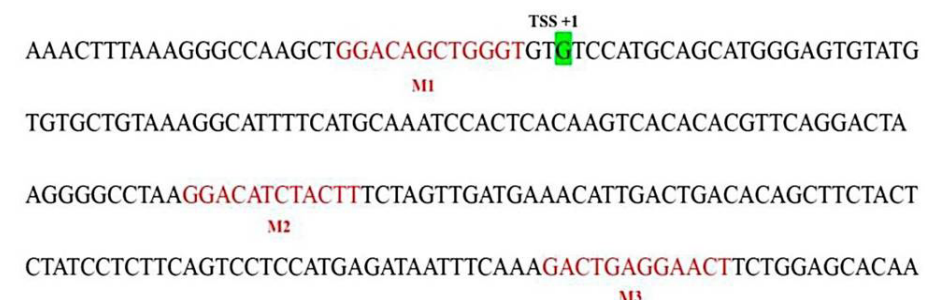

MI3

AGGATAATTTCTCATTGATAAGCCTTCAGTGTTTCTATTTGAGTTGTGTCTACTGGCCCT

CCACCATG
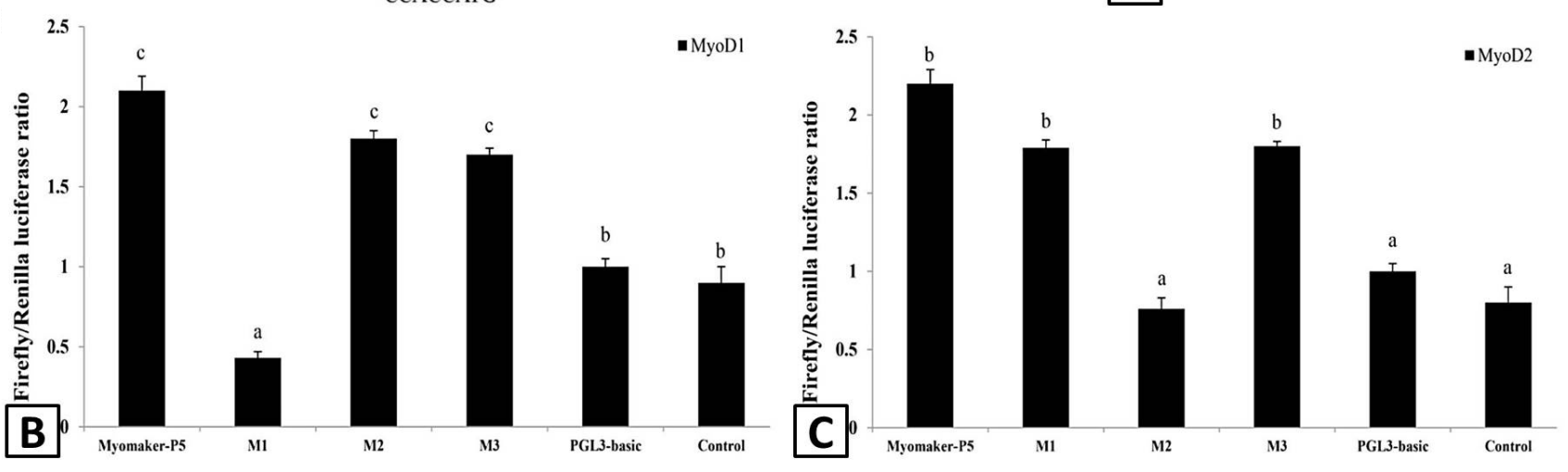

Fig. 6. Construction of truncated mutants for the identification of predicted transcription factor (TF) binding sites in the Almyomaker candidate sequence. A, the nucleotide sequence and predicted binding sites in the core region of the Almyomaker-p5. TSS indicates the transcription start site. Effects of three mutants on Almyomaker-p5 promoter activity transfected with pcDNA3.1-MyoD1 (B) or pcDNA3.1-MyoD2 (C) or pcDNA3.1. Binding sites are shown with boxes. Data are presented as the means of three replicates \pm SE. Different letters indicate significant differences $(p<0.05)$.

Two AlMyoDs activate Almyomaker expression

The amplified candidate Almyomaker promoter and
5'-UTR region (2,090 bp) are upstream nontranslational sequences. To comprehend the binding region of two MyoDs 
in the Almyomaker sequence, a full-length target fragment and 4 truncated mutants were inserted with a promoterless luciferase reporter vector, pGL3-basic (Promega, USA). The result of promoter activity analysis shows that the construct of myomaker-p5 ( $-34 \mathrm{bp}$ to $+267 \mathrm{bp})$ is higher than that of other constructs with cotransfection of MyoD1 or MyoD2, suggesting that the core binding region is located at $-34 \mathrm{bp}$ to $+267 \mathrm{bp}$, which may include several MyoD1 or MyoD2 binding sites (Fig. 5). Therefore, the sequence of construct myomaker-p5 ( -34 bp to $+267 \mathrm{bp}$ ) was used for further functional analysis.

To understand the MyoD1 and MyoD2 binding sites in Almyomaker, the presumptive binding sites are mutated (Fig. 6A; Table II). The effects on promoter activity were investigated in 293T cells, together transfected with each mutant and MyoD1 or MyoD2. The results reveal that mutation of the M1 binding site (GGACAGCTGGGT, $-14 \mathrm{bp}$ to $-3 \mathrm{bp}$ ) generates a marked reduction in promoter activity (Fig. 6B), showing that M1 is the MyoD1 binding site on the Almyomaker promoter. Moreover, mutation of the M2 binding site (GGACATCTACTT, +92 bp to +103 bp) resulted in a significant reduction in promoter activity (Fig. 6C), suggesting that M2 is the potential MyoD2 binding site in the Almyomaker gene 5'-UTR region. Notably, one predicted binding site (M3) does not activate luciferase activity with MyoD1 or MyoD2, suggesting that this site is not required for triggering Almyomaker expression with two MyoDs.

\section{DISCUSSION}

The present study investigated the potential mechanisms of the transcriptional regulation of myomaker by MyoD1 and MyoD2 in A. latus. The sequence and functional characterization, tissue expression and regulation relationship between two AlMyoDs and Almyomaker were determined. The Almyomaker aa sequence was $54.7 \%$ $92.0 \%$ identical to myomaker proteins from other teleosts.

In general, the expression of several genes depends on RNA polymerases and TFs binding to specific sequences on the promoters of target genes in eukaryotic organisms (O’Malley, 1977; Xie et al., 2018; Zhu et al., 2019). Furthermore, the integrity and activity of a promoter could regulate gene transcription. The TF MyoDs monitored skeletal muscle growth in mammals and altered the transcription of muscle-related genes in teleosts and avians (Millay et al., 2014; Luo et al., 2015). Notably, evidence has demonstrated that overexpression of MyoD could increase downstream gene myomaker expression in zebrafish (Bi et al., 2017; Quinn et al., 2017; Zhang et $a l ., 2017)$. It was unclear whether MyoD could upregulate
Almyomaker in marine fishes. In the present study, dualluciferase reporter assays were conducted to realize regulatory mechanisms whereby MyoD1 and MyoD2 were believed to accommodate Almyomaker expression. The results of truncated mutants indicated that Almyomaker reporter activity was activated by the overexpression of two AlMyoDs. The core binding region in the Almyomaker promoter was -34 to +267 bp (Fig. 6). This evidence was the first to show that the expression of Almyomaker could be upregulated by both AlMyoD1 and AlMyoD2 in marine fishes.

MyoDs possess a highly conserved bHLH domain, which is necessary for heterodimerization with characteristic DNA binding to the e-box motif (consensus CANNTG) detected in the regulatory regions of their target genes (Murre et al., 1989; Lassar et al., 1989, 1991; Davis and Weintraub, 1992). Consequently, to investigate the two MyoD binding sites on the Almyomaker promoter, three mutant vectors were constructed. Mutation of the MyoD1 M1 binding site (GGACAGCTGGGT) and MyoD2 M2 binding site (GGACATCTACTT) resulted in observably decreased promoter activity (Fig. 6B, C), suggesting that the MyoD1 binding M1 site and MyoD2 binding M2 site were essential for Almyomaker promoter activity, respectively. The sequences of the above two binding sites were representative e-box motifs (Davis and Weintraub, 1992). In brief, MyoD1 and MyoD2 could mediate myomaker promoter expression by combining e-box motifs (M1 and M2 binding sites) in fish, respectively, which was consistent with the findings in M. musculus and G. gallus (Millay et al., 2014; Luo et al., 2015). Moreover, the MyoD2 M2 binding site was located in the 5'UTR, which was analogous to previous studies (Wu et al., 2018; Khan et al., 2019).

Phylogenetic analysis of myomakers in tetrapods and teleosts showed that a protein of uniform length (221 aa) was observed in ancestral nonteleost fish, and teleost myomaker sequences were classified into three groups according to myomaker protein length (Landemaine et al., 2019). In the present study, phylogenetic analysis showed that a typical phylogeny revealing the amino acid sequence of Almyomaker was closely matched to myomakers of $O$. niloticus, X. maculatus, O. latipes, P. formosa, and T. nigroviridis, with analogous lengths. This result was similar to that reported in Landemaine et al. (2019). A genome structure analysis showed that all myomakers contained 6 exons and 5 introns in metazoans, except for $D$. rerio, $T$. nigroviridis, $P$. sinensis, L. chalumnae, $G$. gallus, H. sapiens and M. musculus myomaker, which only possessed 5 exons and 4 introns, suggesting that the last exon might be lost during evolution.

Previous studies have focused on the role of myomaker 
during embryonic development and have determined that myomaker was indispensable for the facilitation of myoblast fusion in such species as D. rerio, G. gallus, and M. musculus (Millay et al., 2013; Landemaine et al., 2014; Luo et al., 2015; He et al., 2017; Zhang and Roy, 2017). The mRNA expression patterns of the myomaker gene during 8 different postnatal developmental stages in the Japanese flounder (Paralichthys olivaceus) showed that the expression of myomaker at $180 \mathrm{dph}$ was higher than that at other periods (Huang et al., 2019). Moreover, myomaker is expressed only in skeletal muscle in $M$. musculus, G. gallus and Oncorhynchus mykiss (Millay et al., 2013; Landemaine et al., 2019). In the present study, the tissue-specific expression pattern revealed that the highest Almyomaker mRNA expression was detected in white muscle, showing that Almyomaker played an important role in muscle development. Almyomaker is also broadly expressed in other tissues; however, in mammals, chicks and fish, Myomaker is nearly undetectable in other organs (Millay et al., 2013; Landemaine et al., 2019). This discrepancy may be attributable to species diversity.

\section{CONCLUSION}

In conclusion, the full-length Almyomaker genome sequence was cloned. The tissue expression profile indicated that the mRNA level of Almyomaker was highest in the heart and gonad among the detected tissues. The luciferase activity analysis showed that the region from $-34 \mathrm{bp}$ to $+267 \mathrm{bp}$ includes the core binding region. Mutation analyses indicated that the activity of the Almyomaker promoter significantly decreased after the targeted mutation of the M1 and M2 binding sites with MyoD1 and MyoD2, respectively. This study mainly focused on the transcription of Almyomaker by MyoDs in heterologous cells. In the future, more attention should be paid to the mRNA or protein levels of Almyomaker after overexpression of MyoDs in endogenous cells.

\section{ACKNOWLEDGMENTS}

The study was supported by the Natural Science Foundation of Guangdong Province (2017A030310594), the National Infrastructure of Fishery Germplasm Resources Project (2019DKA30470) and the Science and Technology Infrastructure Construction Project of Guangdong Province (2019B030316030).

\section{Supplementary material}

There is supplementary material associated with this article. Access the material online at: https://dx.doi. org/10.17582/journal.pjz/20200116020138
Statement of conflict of interests

The authors declare no competing interests.

\section{REFERENCES}

Berkes, C.A. and Tapscott, S.J., 2005. MyoD and the transcriptional control of myogenesis. Semin. Cell Dev. Biol., 16: 585-595. https://doi.org/10.1016/j. semcdb.2005.07.006

Bi, P.P., Ramirez-Martinez, A., Li, H., Cannavino, J., McAnally, J.R., Shelton, J.M., Sanchez-Ortiz, E., Bassel-Duby, R. and Olson, E.N., 2017. Control of muscle formation by the fusogenic micropeptide myomixer. Science, 356: 323-327. https://doi. org/10.1126/science.aam9361

Bologna, G., Yvon, C., Duvaud, S. and Veuthey, A.L., 2004. N-Terminal myristoylation predictions by ensembles of neural networks. Proteomics, 4: 16261632. https://doi.org/10.1002/pmic.200300783

Braun, T., Buschhausen-Denker, G., Bober, E., Tannich, E. and Arnold, H.H., 1989. A novel human muscle factor related to but distinct from MyoD1 induces myogenic conversion in 10T1/2 fibroblasts. $E M B O J$, , 8: 701-709. https://doi. org/10.1002/j.1460-2075.1989.tb03429.x

Braun, T. and Gautel, M., 2011. Transcriptional mechanisms regulating skeletal muscle differentiation, growth and homeostasis. Nat. Rev. Mol. Cell Biol., 12: 349-361. https://doi. org/10.1038/nrm3118

Charrin S., Latil M., Soave S., Polesskaya A., Christien F., Boucheix C. and Rubinstein E., 2013. Normal muscle regeneration requires tight control of muscle cell fusion by tetraspanins CD9 and CD81. Nat. Commun., 4: 1674. https://doi.org/10.1038/ ncomms 2675

Davis, R.L. and Weintraub, H., 1992. Acquisition of myogenic specificity by replacement of three amino acid residues from MyoD into E12. Science, 256: 1027-1030. https://doi.org/10.1126/ science. 1317057

Demonbreun, A.R., Biersmith, B.H. and Mcnally, E.M., 2015. Membrane fusion in muscle development and repair. Semin. Cell Dev. Biol., 45: 48-56. https:// doi.org/10.1016/j.semcdb.2015.10.026

Doherty, K.R., Cave, A., Davis, D.B., Delmonte, A.J., Posey, A. and Earley, J.U., 2005. Normal myoblast fusion requires myoferlin. Development, 132: 5565-5575. https://doi.org/10.1242/dev.02155

Edmondson, D.G. and Olson, E.N., 1990. A gene with homology to the myc similarity region of MyoD1 is expressed during myogenesis and is sufficient to 
activate the muscle differentiation program. Genes Dev., 4: 1450. https://doi.org/10.1101/gad.4.8.1450

Ganassi, M., Badodi, S., Quiroga, H.P.O., Zammit, P.S., Hinits, Y. and Hughes, S.M., 2018. Myogenin promotes myocyte fusion to balance fibre number and size. Nat. Commun., 9: 4232. https://doi. org/10.1038/s41467-018-06583-6

He, K., Ren, T., Zhu, S., Liang, S. and Zhao, A., 2017. Transiently expressed pattern during myogenesis and candidate miRNAs of Tmem8C in goose. $J$. Genet., 96: 39-46. https://doi.org/10.1007/s12041016-0737-8

Hinits, Y. and Hughes, S.M., 2007. Mef2s are required for thick filament formation in nascent muscle fibres. Development, 134: 2511-2519. https://doi. org $/ 10.1242 /$ dev. 007088

Huang, Y., Wu, S., Zhang, J., Wen, H., Zhang, M. and He, F., 2019. Methylation status and expression patterns of myomaker gene play important roles in postnatal development in the Japanese flounder (Paralichthys olivaceus). Gen. Comp. Endocrinol., 280: 104-114. https://doi.org/10.1016/j.ygcen.2019.04.017

Khan, R., Raza, S.H.A., Junjvlieke, Z., Wang, X.Y., Garcia, M., Elnour, I.E., Wang H.B. and Zan, L.S., 2019. Function and transcriptional regulation of bovine TORC2 gene in adipocytes: Roles of $\mathrm{C} /$ EBP $\gamma$, XBP1, INSM1 and ZNF263. Int. J. Mol. Sci., 20: 4338. https://doi.org/10.3390/ijms20184338

Kim, J.H., Jin, P., Duan, R. and Chen, E.H., 2015. Mechanisms of myoblast fusion during muscle development. Curr. Opin. Genet. Dev., 32: 162170. https://doi.org/10.1016/j.gde.2015.03.006

Landemaine, A., Rescan, P.Y. and Gabillard, J.C., 2014. Myomaker mediates fusion of fast myocytes in zebrafish embryos. Biochem. biophys. Res. Commun., 451: 480-484. https://doi.org/10.1016/j. bbrc.2014.07.093

Landemaine, A., Ramirez-Martinez, A., Monestier, O., Sabin, N., Rescan, P.Y., Olson, E.N. and Gabillard, J.C., 2019. Trout myomaker contains 14 minisatellites and two sequence extensions but retains fusogenic function. J. biol. Chem., 294: 6364-6374. https://doi.org/10.1074/jbc. RA118.006047

Larkin, M.A., Blackshields, G., Brown, N.P. and Higgins, D.G., 2007. ClustalW and ClustalX version 2. Bioinformatics, 23: 2947-2948. https:// doi.org/10.1093/bioinformatics/btm404

Lassar, A.B., Buskin, J.N., Lockshon, D., Davis, R.L., Apone, S., Hauschka, S.D. and Weintraub, H., 1989. MyoD is a sequence specific DNA binding protein requiring a region of myc homology to bind to the muscle creatine kinase enhancer. Cell, 58: 823-831. https://doi.org/10.1016/0092-8674(89)90935-5

Lassar, A.B., Davis, R.L., Wright, W.E., Kadesch, T., Murre, C., Vorona, A., Baltimore, D. and Weintraub, H., 1991. Functional activity of myogenic HLH proteins requires hetero-oligomerization with E12/ E47-like proteins in vivo. Cell, 66: 305-315. https:// doi.org/10.1016/0092-8674(91)90620-E

Li, S.L., Monroig, O., Wang, T.J., Yuan, Y.H., Navarro, J.C., Hontoria, F., Liao, K., Tocher, D.R., Mai, K.S. and $\mathrm{Xu}, \mathrm{W} ., 2017$. Functional characterization and differential nutritional regulation of putative Elovl5 and Elovl4 elongases in large yellow croaker (Larimichthys crocea). Scient. Rep., 7: 2303. https://doi.org/10.1038/s41598-017-02646-8

Livak, K.J. and Schmittgen, T.D., 2001. Analysis of relative gene expression data using real-time quantitative PCR and the $2^{-\Delta \Delta C t}$ method. Methods, 25: 402-408. https://doi.org/10.1006/meth.2001.1262

Luo, W., Li, E., Nie, Q.H. and Zhang, X.Q., 2015. Myomaker, Regulated by MyoD, MyoG and miR140-3p, Promotes chicken myoblast fusion. Int. J. mol. Sci., 16: 26186-26201. https://doi.org/10.3390/ ijms 161125946

Millay, D.P., Gamage, D.G., Quinn, M.E., Min, Y.L., Mitani, Y., Bassel-Duby, R. and Olson, E.N., 2016. Structure-function analysis of myomaker domains required for myoblast fusion. Proc. natl. Acad. Sci. U.S.A., 113: 2116-2121. https://doi.org/10.1073/ pnas. 1600101113

Millay, D.P., O'Rourke, J.R., Sutherland, L.B., Bezprozvannaya, S., Shelton, J.M., Bassel-Duby, R. and Olson, E.N., 2013. Myomaker is a membrane activator of myoblast fusion and muscle formation. Nature, 499: 301-305. https://doi.org/10.1038/ nature 12343

Millay, D.P., Sutherland, L.B., Bassel-Duby, R. and Olson, E.N., 2014. Myomaker is essential for muscle regeneration. Genes Dev., 28: 1641-1646. https://doi.org/10.1101/gad.247205.114

Murre, C., McCaw, P.S., Vaessin, H., Caudy, H., Jan, L.Y., Jan, Y.N., Cabrera, C.V., Buskin, J.N., Hauschka, S.D., Lassar, A.B., Weintraub, H. and Baltimore, D., 1989. Interactions between heterologous helix-loop-helix proteins generate complexes that bind specifically to a common DNA sequence. Cell, 58: 537-544. https://doi. org/10.1016/0092-8674(89)90434-0

O'Malley, B.W., Towle, H.C. and Schwartz, R.J., 1977. Regulation of gene expression in eucaryotes. Annu. Rev. Genet., 11: 239-275. https://doi.org/10.1146/ annurev.ge.11.120177.001323 
Powell, G.T. and Wright, G.J., 2011. Jamb and Jamc are essential for vertebrate myocyte fusion. PLoS Biol., 9: e1001216. https://doi.org/10.1371/journal. pbio. 1001216

Quinn, M.E., Goh, Q.N., Kurosaka, M., Gamage, D.G., Petrany, M.J., Prasad, V. and Millay, D.P., 2017. Myomerger induce fusion of non-fusogenic cells and is required for skeletal muscle development. Nat. Commun., 8: 15665. https://doi.org/10.1038/ ncomms 15665

Shi, J., Cai, M.X., Si, Y.F., Zhang, J.S. and Du, S.J., 2018. Knockout of myomaker results in defective myoblast fusion, reduced muscle growth and increased adipocyte infiltration in zebrafish skeletal muscle. Hum. Mol. Genet., 27: 3542-3554. https:// doi.org/10.1093/hmg/ddy268

Si, Y.F., Wen, H.S. and Du, S.J., 2019. Genetic mutations in jamb, jamc, and myomaker revealed different roles on myoblast fusion and muscle growth. Mar. Biotechnol., 21: 111-123. https://doi.org/10.1007/ s10126-018-9865-x

Sohn, R.L., Huang, P., Kawahara, G., Mitchell, M., Guyon, J., Kalluri, R., Kunkel, L.M. and Gussoni, E., 2009. A role for nephrin, a renal protein, in vertebrate skeletal muscle cell fusion. Proc. natl. Acad.Sci., 106: 9274-9279. https://doi.org/10.1073/ pnas.0904398106

Sun, L.Y., Zhang, D.C., Jiang, S.G., Guo, H.Y. and Zhu, C.Y., 2013. Isolation and characterization of 21 polymorphic microstatellites in golden pompano Trachinotus ovatus. Conserv. Genet. Resour., 5: 1107-1109. https://doi.org/10.1007/s12686-0139942-4

Tamura, K., Stecher, G., Peterson, D., Filipski, A. and Kumar, S., 2013. MEGA6: Molecular evolutionary genetics analysis, Version 6.0. Mol. Biol., 3: 2725 2729. https://doi.org/10.1093/molbev/mst197

Vasyutina, E., Martarelli, B., Brakebusch, C., Wende, H. and Birchmeier, C., 2009. The small G-proteins Rac1 and Cdc42 are essential for myoblast fusion in the mouse. Proc. natl. Acad. Sci., 106: 89358940. https://doi.org/10.1073/pnas.0902501106

Wu, X., Xu, F.L., Ding M., Zhang, J.J., Yao, J. and Wang, B.J., 2018. Characterization and functional analyses of the human HTR1A gene: 5' regulatory region modulates gene expression in vitro. $B M C$ Genet., 19: 115. https://doi.org/10.1186/s12863018-0708-6.

Xie, D., Fu, Z., Wang, S., You, C., Monroig, Ó., Tocher, D.R. and Li, Y., 2018. Characteristics of the fads2 gene promoter in marine teleost Epinephelus coioides and role of Sp1-binding site in determining promoter activity. Scient. Rep., 8: 5305. https://doi. org/10.1038/s41598-018-23668-w

Tapscott, S.J., 2005. The circuitry of a master switch: Myod and the regulation of skeletal muscle gene transcription. Development, 132: 2685-2695. https://doi.org/10.1242/dev.01874

Zhang, Q., Vashisht, A.A., O’Rourke, J., Corbel, S.Y., Moran, R., Romero, A., Miraglia, L., Zhang, J., Durrant, E. and Schmedt, C., 2017. The microprotein Minion controls cell fusion and muscle formation. Nat. Commun., 8: 15664. https://doi.org/10.1038/ ncomms 15664

Zhang, W.B. and Roy, S., 2017. Myomaker is required for the fusion of fast-twitch myocytes in the zebrafish embryo. Dev. Biol., 423: 24-33. https:// doi.org/10.1016/j.ydbio.2017.01.019

Zhu, K.C., Chen, L.P., Zhao, J.K., Wang, H.J., Wang, W.M., Li, Z. and Wang, H.L., 2014. Molecular characterization and expression patterns of myogenin in compensatory growth of Megalobrama amblycephala. Comp. Biochem. Physiol. B, 170: 10-17. https://doi.org/10.1016/j.cbpb.2014.01.001

Zhu, K.C., Song, L., Guo, H.Y., Guo, L., Zhang, N., Liu, B.S., Jiang, S.G. and Zhang, D.C., 2019. Elov14a participates in LC-PUFA biosynthesis and is regulated by PPAR $\alpha \beta$ in golden pompano Trachinotus ovatus (Linnaeus 1758). Scient. Rep., 9: 4684. https://doi.org/10.1038/s41598-01941288-w

Zhu, K.C., Liu, B.S., Guo, H.Y., Zhang, N., Guo, L., Jiang, S.G. and Zhang, D.C., 2020. Functional analysis of two MyoDs revealed their role in the activation of myomixer expression in yellowfin seabream (Acanthopagrus latus) (Hottuyn, 1782). Int. J. Biol. Macromol., https://doi.org/10.1016/j. ijbiomac.2019.11.139 